\title{
Experimental Study on the Biaxial Te sile Properties of Two Bar Warp-knit Fabrics
}

\author{
By Yoshiki Yanagawa*, Sueo Kawabata** and Hiromichi Kawai**, Members, TMSJ \\ *Department of Textile Technology, Kyoto University of Industrial Arts and Textile Fibers, Kyoto \\ **Department of Polymer Chemistry, Kyoto University, Kyoto \\ Based on the Journal of the Textile Machinery Society of Japan, Transactions, Vol. 24, No. 12, T223-231 (1971)
}

\begin{abstract}
In the previous paper, a theoretical method for calculating the biaxial tensile property of two bar tricot fabrics had been presented.

In this paper, the accuracy of this theory has teen investigated by measuring the tensile properties of some actual fabrics by means of a biaxial tensile tester, and comparing the measured values with the theoretical ones.

The result shows that the fabrics knitted of fine or low-bulky yarns provide a very good agreement between the theoretical and experimental results. For the fabrics knitted of thick or high-bulky yarns, however, there is some discrepancy between them in some biaxial-deformation modes.

Two improvements have been done to this theory based on those observations: (1) the structure of the unit model has been corrected to be closer to the actual fabric structure, and (2) the compressive deformations of yarns in the needle and the sinker loops are calculated separately.

These improvements have made to the theory more useful for the practical design of almost all kinds of two bar tricot fabrics.
\end{abstract}

KEY WORDS: WARP KNITTED FABRICS, TRICOT KNITTED FABRICS, TRICOT STITCHES, FABRIC STRUCTURE, BENDING, TENSILE PROPERTIES

\section{Introduction}

In the previous paper ${ }^{[1]}$, the theoretical analysis of the biaxial-tensile property of two-bar tricot fabrics was presented. In this paper, the accuracy of this theory is examined by comparing the experimental values with the theoretical ones on some actual fabrics.

Because some discrepancy between the theoretical and the experimental values has been found in the case of fabrics knitted of thick or high-bulky yarns, the theory is partly corrected on the basis of experimental results. At the same time, the structural model offered in the previous paper $^{[1]}$ is also examined by a microscopic method.

\section{Experimental Apparatus and Method}

The biaxial tensile property of some actual fabrics was measured by using the biaxial tensile tester ${ }^{[2]}$, the detail of which have been already explained in earlier papers ${ }^{[3,4,5]}$.

The frictional, bending and compressive properties of yarns, required for theoretical calculation, were obtained as follows:
The coeffecient $\mu$, of friction between yarns, was calculated by

$$
\mu=\log _{10}\left(\left[T_{1}\right] /\left[T_{2}\right]\right) / 0.68218
$$

where $\left[T_{1}\right]$ and $\left[T_{2}\right]$ are leaving and incoming tensions respectively when a yarn passes vertically with contact angle of $90^{\circ}$ around another yarn which is spanned horizontally.

With filament yarns, their bending rigidity was calculated by summing up the rigidity of each single filament which was obtained from each Young's modulus and its cross-sectional shape. As for spun yarns, the rigidity was measured by the ring. yarn method presented by Kawabata et al. ${ }^{[6]}$, in which many loops of a yarn are pulled with knife edge by the tensile tester, and the relation between the tensile load and the elongation is measured. Then, the bending rigidity of the yarn is calculated by the elastica theory of bended thin rods ${ }^{[6]}$.

The compressive property of a yarn was measured by the apparatus proposed by Kawabata ${ }^{[7]}$, in which a yarn pulled with a given tension is compressed between two plates. The resolving power of the apparatus is $0.2 \mu^{[7]}$. 


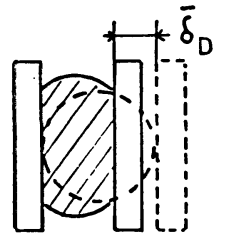

(a)

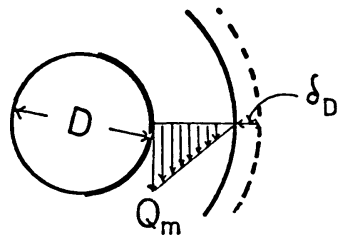

(b)
Fig. 1 Measurement of compresssive property and compressive deformation of yarn in crossover region

In this theory, the diameter, Dc, of the yarn in the closest packing state, is used as the yarin thickness at the critical tensile state. From this reason, the compressive property of a yarn must be measured in a stretched state, that is, in the closest packing state. The elongation of the yarn to reach this state is from 5 to $10 \%$, depending on the yarn structure, and must be decided by experiment.

The compressed deformation measured by this apparatus is the value by the parallel plate method as shown in Fig. 1 (a). However, the pressure on the yarn is actually caused by fiber tension, and the pressure distribution in the cross section of the yarn will be as shown in Fig. 1(b). In the model described in the previous paper ${ }^{[1]}$, in which a yarn having of tension $T$ is wound around a rod with diameter $D$, it is considered that the maximum pressure $Q_{m}$ is found at the contact surface and given by

$$
Q_{m}=2 T / D
$$

and the pressure at the outer layer becomes zero. Therefore, $\bar{\delta}_{D}$, the deformation measured by the parallel plate method, is larger than $\delta_{D}$, the deformation actually found in yarn-to-yarn contact, and should be corrected by taking this pressure distribution into consideration.

It is assumed that all fibers in the cross-section of the yarn have equal tension, and that the pressure caused by the tension changes linearly from the outer layer to the contact layer in the yarn. Then, the relation between $\bar{\delta}_{D}$ and $\delta_{D}$ is expressed by the formula introduced by Kawabata et al. ${ }^{[6]}$ as follows :

$$
-\frac{\delta \mathrm{D}}{\delta_{\mathrm{D}}}=\frac{1-\frac{1}{\alpha}\left(1-e^{-\alpha}\right)}{1-e^{-\alpha}}
$$

where $\alpha=k Q_{m}$, and $k$ is a constant characterizing the compressive property of the yarn ${ }^{[6]}$.

The measured values of compressive property of the yarn are corrected here by using eq. (3). The propriety of the corrected will be discussed later.

\section{Specimens}

The structural constants of the specimens, the frictional coefficient and the bending rigidity of the yarn used here are shown in Table 1. The tensile properties are shown in

\begin{tabular}{|c|c|c|c|c|c|c|c|c|c|}
\hline \multicolumn{2}{|c|}{ Specimen No. } & $\$ 1$ & $\# 2$ & $\# 3$ & $\# 4$ & \#5 & $\# 6$ & $\$ 7$ & $\$ 8$ \\
\hline \multicolumn{2}{|l|}{ Stitch } & half & half & $\begin{array}{l}\text { back } \\
\text { half }\end{array}$ & $\begin{array}{l}\text { satin } \\
\text { back }\end{array}$ & sharkskin & half & half & half \\
\hline \multicolumn{2}{|l|}{$n_{f}$} & 2 & 2 & 1 & 3 & 1 & 2 & 2 & 2 \\
\hline \multicolumn{2}{|l|}{$n_{b}$} & 1 & 1 & 2 & 1 & 3 & 1 & 1 & 1 \\
\hline \multicolumn{2}{|c|}{ Course spacing $(\mathrm{mm})$} & 0.435 & 0.559 & 0.535 & 0.594 & 0.591 & 0.660 & 0.781 & 0.661 \\
\hline \multicolumn{2}{|c|}{ Wale spacing $(\mathrm{mm})$} & 0.558 & 0.670 & 0.848 & 0.669 & 0.818 & 1.058 & 1.074 & 1.118 \\
\hline \multicolumn{2}{|c|}{$\begin{array}{l}\text { Length of unit structure } \\
\qquad(\mathrm{mm})\end{array}$} & 5.491 & 6.782 & 6.394 & 7.925 & 8.384 & 9.377 & 10.265 & 9.784 \\
\hline \multirow{7}{*}{ 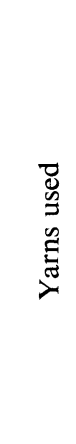 } & Materials & nylon & $\begin{array}{l}\text { poly- } \\
\text { propylene }\end{array}$ & polyester & nylon & nylon & polyester & acrylic & cotton \\
\hline & Yarn count & 30 den & 40 den & 50 den & 100 den & 100 den & 30 's & 29.01 's & 31.27 's \\
\hline & $\begin{array}{l}\text { Number of } \\
\text { fibers }\end{array}$ & 6 & 12 & 18 & 24 & 24 & 118 & 105 & 93 \\
\hline & $\begin{array}{l}\text { Yarn diameter } \\
D(\mathrm{~mm})\end{array}$ & 0.0598 & 0.0781 & 0.0711 & 0.1108 & 0.1108 & 0.1346 & 0.1494 & 0.1355 \\
\hline & $\begin{array}{l}\text { Tuist of yarn } \\
\text { (turns } / \mathrm{m} \text { ) }\end{array}$ & 7 & 15 & 15 & 10 & 10 & 880 & 730 & 920 \\
\hline & $\begin{array}{l}\text { Bending rigidity } \\
\text { of yarn }\left(\mathrm{g} \cdot \mathrm{mm}^{2}\right)\end{array}$ & 0.0247 & 0.0591 & 0.0596 & 0.0887 & 0.0887 & 0.0613 & 0.1081 & 0.0646 \\
\hline & $\begin{array}{l}\text { Coefficient of } \\
\text { friction }(\mu)\end{array}$ & 0.1686 & 0.1415 & 0.1780 & 0.2201 & 0.2201 & 0.2848 & 0.2890 & 0.3374 \\
\hline
\end{tabular}

Table 1 Specimens 


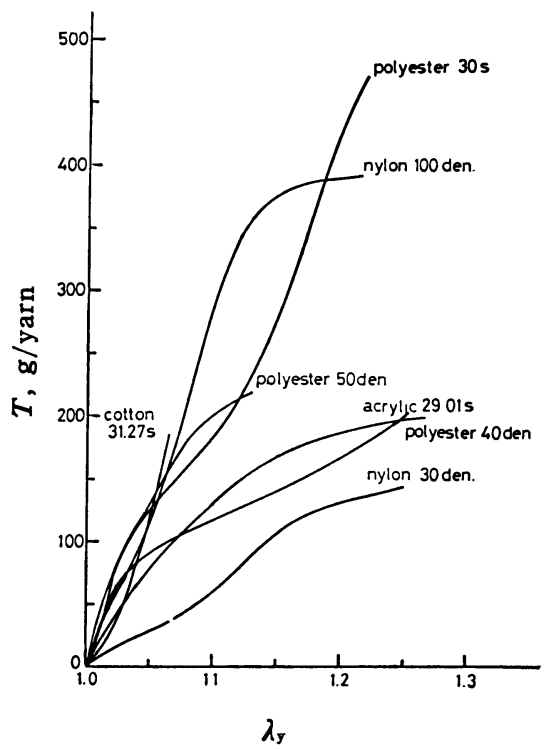

Fig. 2 Tensile property of yarn

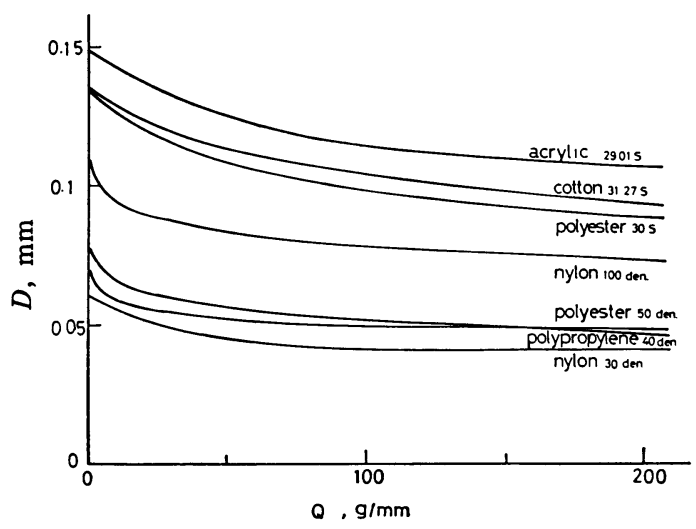

Fig. 3 Compressive property of yarn

Fig. 2, the compressive property in Fig. 3. The stitches of these specimens are half, back half, sharkskin and back satin, and the yarns are nylon, acrylic, polyester, polypropylene and cotton $100 \%$, respectively. All the fabrics are in grey and applied no finishing treatment.

\section{Results and Discussion}

4.1 The Shape of Stitches in Biaxial Deformation

The structure of stitch in biaxial extension was observed by the microscopic method.

Fig. 4 shows the uniform biaxial deformation of \#5 specimen near breaking point. As seen from the figure, the difference between unit stitch structures is caused only by the number of wales shifted in underlapping, and the fabric has $\left(n_{f}+n_{b}\right)$ ply layers of unit structures, where $n_{f}$ is the number of wales shifted by the front-bar in under-

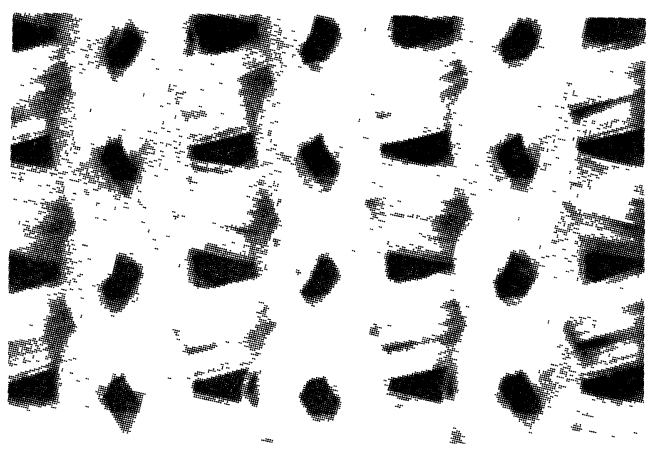

Fig. 4 Stitch structure at high stretch ratios

$\left(\$ 5\right.$ nylon 100 den sharkskin fabric, $\lambda_{1}=\lambda_{2}=1.17$ )

lapping, $n_{b}$ being that by the back-bar. The stru.cture in the critical tensile state ${ }^{[1]}$ can be thought very similar to the structure of the actual deformation.

The cross section of the stitches was examired as shown in Fig. 5. The precise observation of the compressive deformation of the yarn at the contact point caused by biaxial stretching is difficult, because yarns are complicatedly entangled. But it can be observed from this photograph that the flattening of the yarn takes place outside the yarn in the crossover region, and that the yarn wound with the outer yarn is nearly in the closest packing state as assumed in the previous paper ${ }^{[1]}$.

\subsection{Effect of Compressive Property of Yarn}

Fig. 6 shows experimental and theoretical curves of the tensile property of specimen $\# 6$ (half fabric knitted of polyester spun yarn), when $k_{2}=0$, that is, in biaxial extension in strip form, and the ordinate indicates the tensile load per course or wa!e, the abscissa being the stretch ratio. Solid line shows experimental curves, while a dotted line shows the theoretical curve calculated by the theory in the previous paper ${ }^{[1]}$, a chain line being the curve ca'culated on the assumption that the yarn diameter does not change during the tensile deformation.

As shown in this figure, the accuracy of this theory can be remarkably improved by taking the compressive property of yarn into consideration. In this figure, two-dot-dash lines show the curves calculated by using the directly measured values of the compressive property of the yarn.

As the solid line expressing the experimental results is closer to the dotted curve than the two-dot-dash-line, it is evident that the measured values of the compressive property must be corrected by considering the pressure distribution.

\subsection{In the case of the Fabric Knitted of Fine Yarns}

Figs. 7 to 9 show the experimental and theoretical curves of specimen $\sharp 1$, $\# 2$ and $\# 3$, knitted of fine yarns (nylon 30 den, polypropylene 40 den and polyester 50 den). The compressive deformation of yarns is relatively smaller 


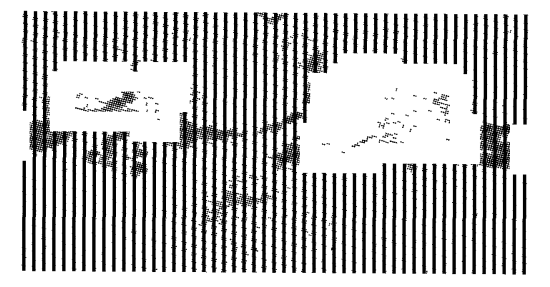

cross section $\mathrm{A}$

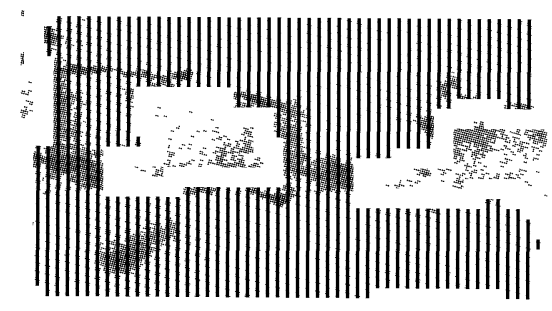

cross section $\mathbf{C}$

Nylon 100 den plain tricot fabric $\left(\lambda_{1}=0.85, \lambda_{2}=1.5\right)$

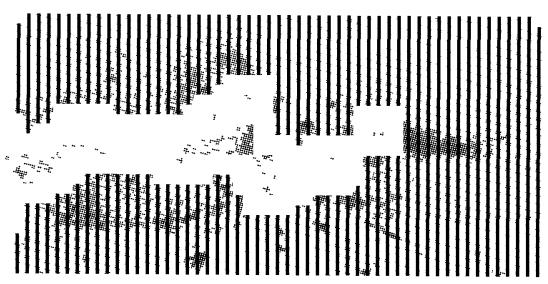

cross section A

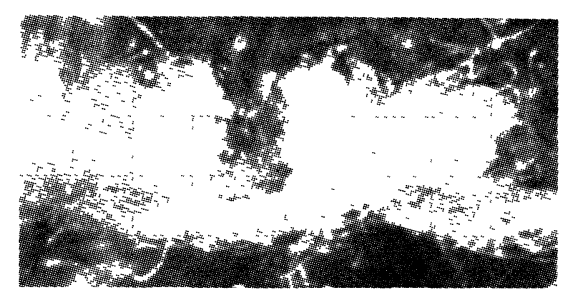

cross section $\mathrm{B}$

Cotton half fabric

$\left(\lambda_{1}=1.25, \lambda_{2}=1.05\right)$

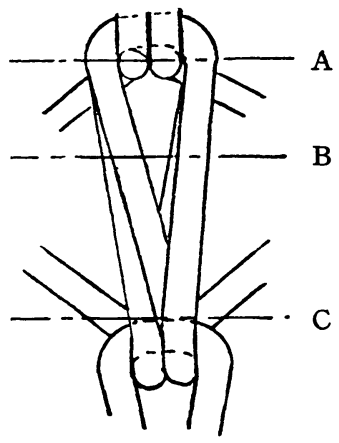

Fig. 5 Cross section of fabric under stretching
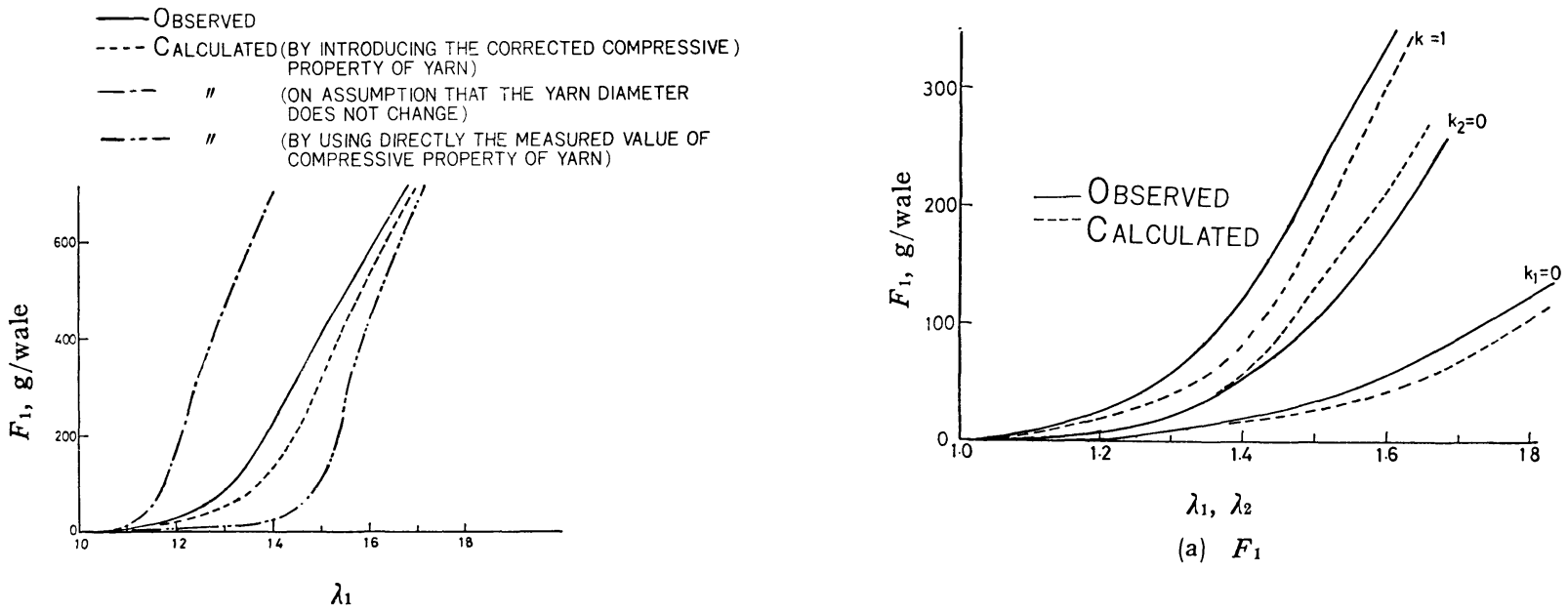

(a) $F_{1}$

(a) $F_{1}$

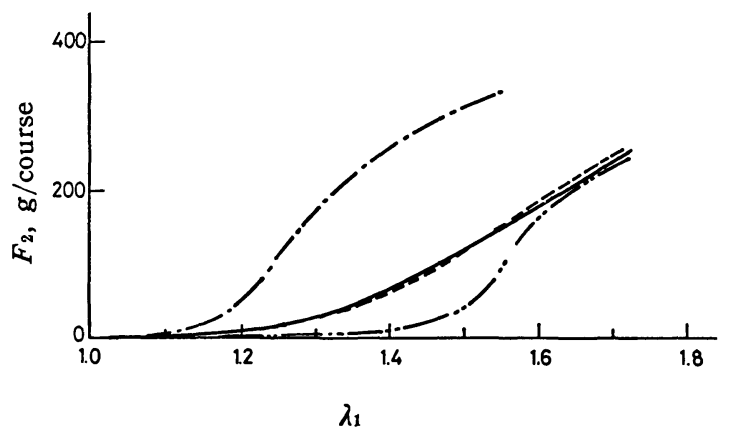

(b) $F_{2}$

Fig. 6 Effect of compressive property of yarn ( $\$ 6$ polyester 30 's half fabric, $K_{2}=0$ )

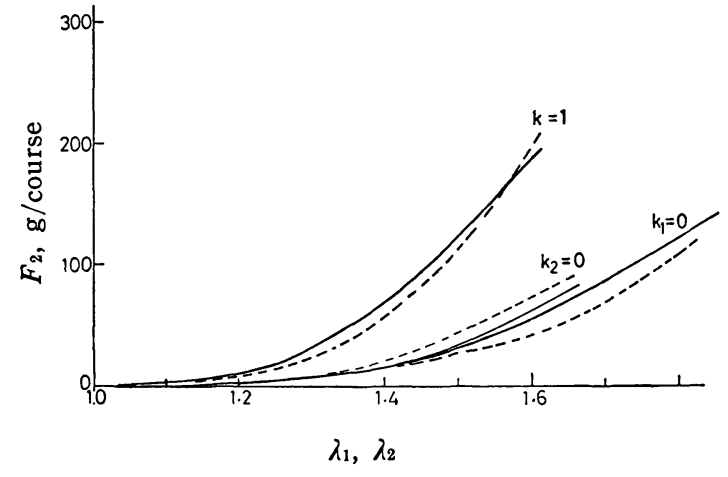

(b) $F_{2}$

Fig. 7 Comparison between theoretical and experimentsal curves ( $\$ 1$ nylon 30 den half fabric)

Journal of The Textile Machinery Society of Japan 


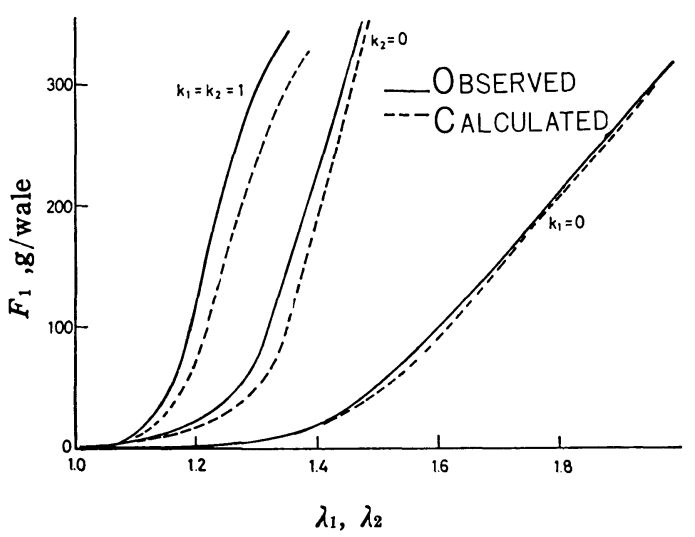

(a) $F_{1}$

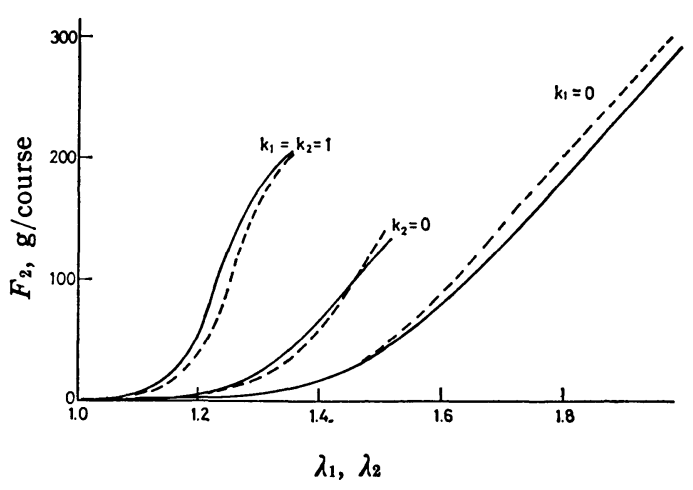

(b) $F_{2}$

Fig. 8 Comparison between theoretical and experimental curves ( $\$ 2$ polypropylene 40 den half fabric)

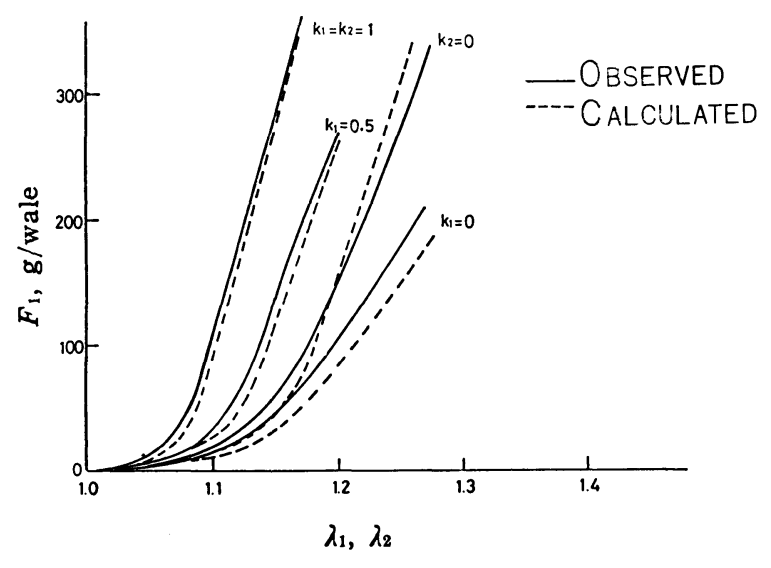

(a) $F_{1}$

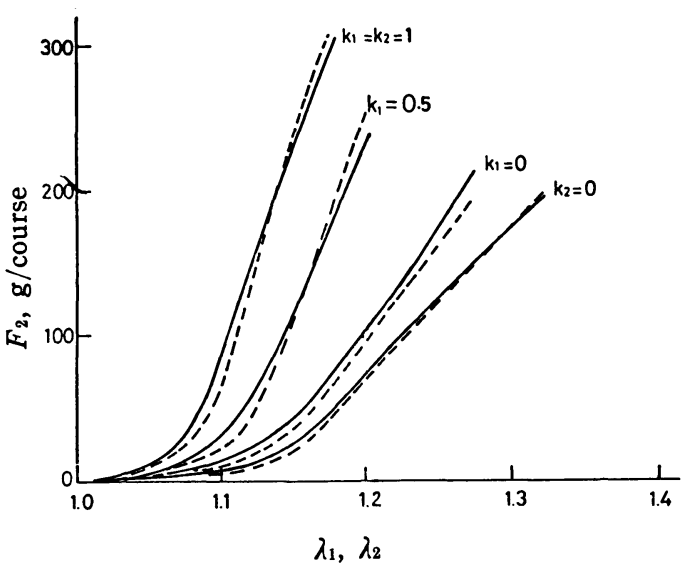

(b) $F_{2}$

Fig. 9 Comparison between theoretical and experimental curves ( $\$ 2$ polyester 50 den back half fabric)

\section{-OBSERVEd}

-..- CALCULATED BY THE IMPROVED THEORY

-- C ALCULATED BY THE THEORY IN THE PREVIOUS PAPER

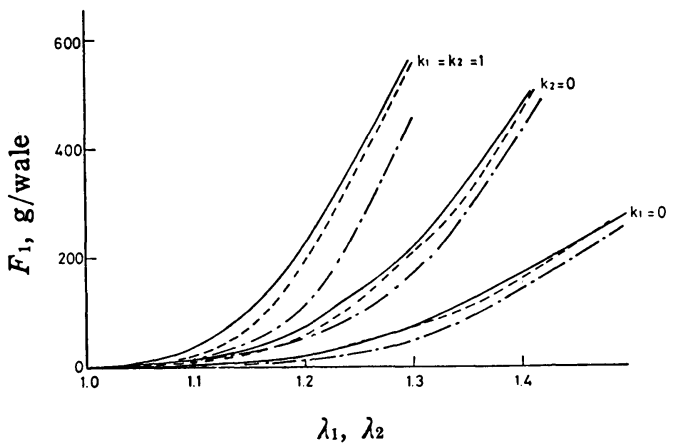

(a) $F_{1}$

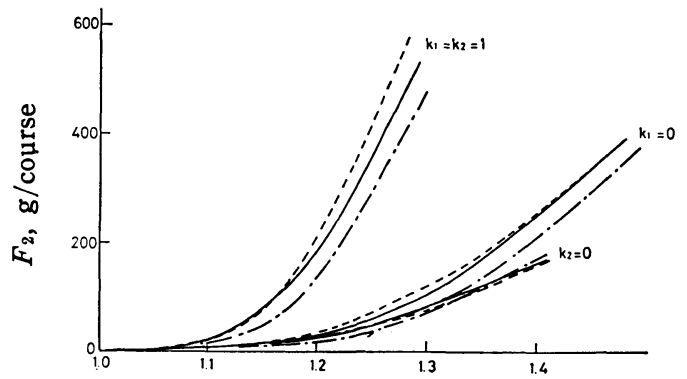

$\lambda_{1}, \lambda_{2}$

Fig. 10 Comparison between theoretical and experimental c rves ( $\$ 4$ nylon 100 den satin back fabric) 
- ObSeRVEd

--- CALCULATED BY THE IMPROVED THEORY

- - CALCULATED BY THE THEORY IN THE PREVIOUS PAPER

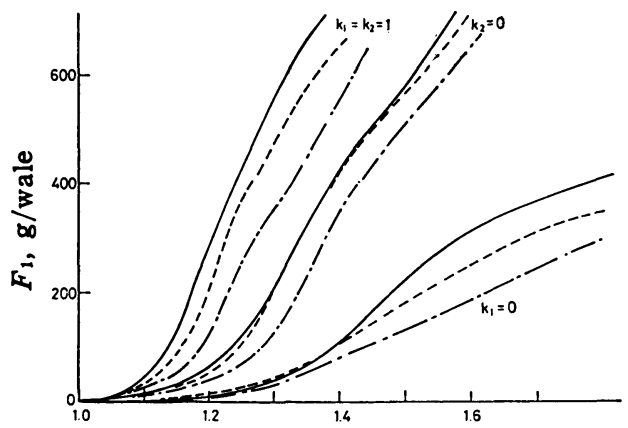

$\lambda_{1}, \lambda_{2}$

(a) $F_{1}$

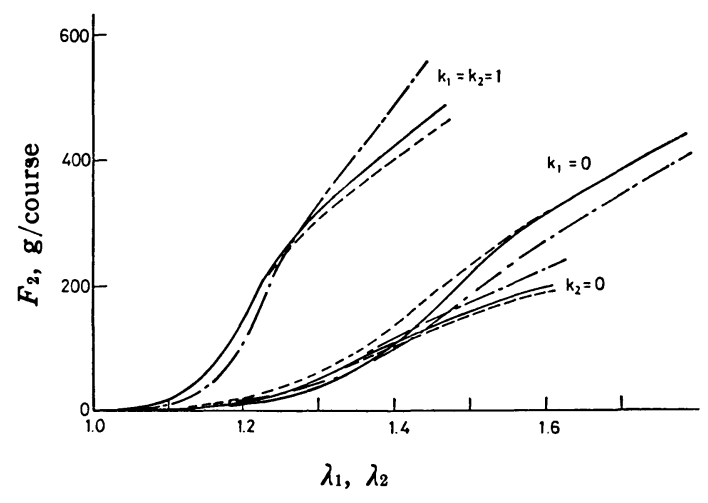

(b) $F_{2}$

Fig. 11 Comparison between theoretical and experimental curves ( $\$ 7$ acrylic 29 's half fabric)

than that obtained with other specimens. Theoretical and experimental values show fairly good agreement.

4.4 In the case of the Fabrics Knitted of High-bulky Yarns

Figs. 10 and 11 show theoretical and experimental curves of $\$ 5$ nylon 100 den back-satin stitch and $\# 7$ acrylic 29 's spun yarn half stitch. In these figures, solid lines indicate experimental results, chain lines the values by the theory in the previous paper ${ }^{[1]}$, dotted lines those by the theory improved as described later.

As is clear from the comparison between solid and chain lines, in the case of biaxial extension in strip form along wale $\left(\mathrm{k}_{2}=0\right)$, fairly good agreement between them is observed for both specimens. But in biaxial extension along course $\left(\mathrm{k}_{1}=0\right)$ and in homogeneous extension $\left(\mathrm{k}_{1}=\mathrm{k}_{2}=1\right)$, there are some disagreements between them. This tendency becomes clear in the fabric knitted of spun yarns having large diameter and high compressibility because of bulkiness. This discrepancy is presumed to be owing to the following two reasons:
One of the reasons is that the theoretical calculation was done for simplicity on the assumption that the compressive deformations of the yarn are the same at all contact points in the crossover region, as described in the previous paper $^{[1]}$. This simplification of the theory makes the error arise, because in the case of the deformation modes abovementioned the degree of the yarn flattening is considerably influenced by the place as follows: As the stretch ratio of the yarn in needle loop, $\lambda_{y m}$, is subjected mainly to $\lambda_{1}$ (the stretch ratio of the fabric along wale direction), on the other hand the stretch ratio of the yarn in sinker loop, $\lambda_{y 1}$, is a function of both stretch ratios $\lambda_{1}$ and $\lambda_{2}$ (stretch ratios along the wale and the course directions respectively). If $\lambda_{2} / \lambda_{1}$ takes larger value, then, there are large differences between yarn tensions of those loops, and the difference between the compressive deformations of both loops becomes larger, because this deformation is proportional to the yarn tension.

So, in the case of the theoretical calculations for thick and high-bulky yarn, this error caused by the difference of compressive deformation tecomes large.

The other reason is the uncertainty of the boundary between needle and sinker loops in the model. As shown in Fig. 12, points $N_{1}$ and $N_{2}$ were placed at the boundary in the model in the stretch-effective region. In the actual structure, however, it is estimated that the force by which two cross-over yarns are pushed against each other at point $f$ in Fig. 12 is not so large that the boundary cannot be clearly decided.

In the fabrics of fine yarns, the boundary becomes clear because the length $\overline{N_{2}^{\prime} f}$ becomes short enough. In the fabrics of thick yarns or hign-bulky yarns, however, this

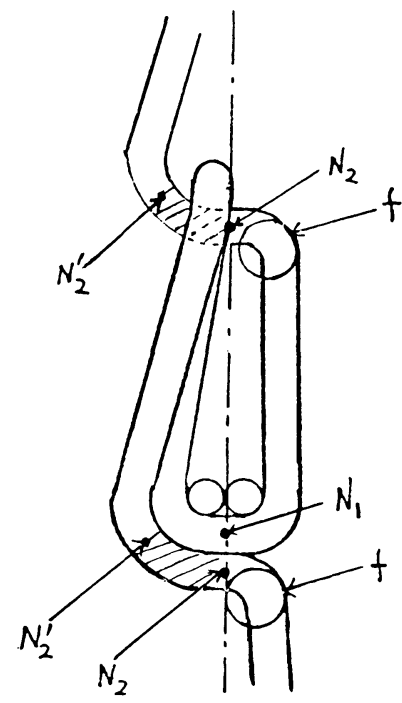

Fig 12 Boundary between needle and sinker loops (Side view of structure front-bar yarn) 
boundary becomes much difficult to identify, and causes error in theoretical calculation.

From these reasons, the theory presented in the previous paper $^{[1]}$ must be improved.

\section{Improvement of Theory}

Assume that points $N_{1}$ and $N_{2}{ }^{\prime}$ be on the boundaries between needle and sinker loops, and that the unit structure terminates at point $N_{2}{ }^{\prime}$, as shown in Fig. 12.

Let $D_{p f s}$ and $D_{p f n}$ be the thicknesses of the flattened yarns of sinker and needle loops respectively at given stretch ratios $\lambda_{s 1}$ and $\lambda_{s 2}$ in stretch effective region. Let $y_{1 c}$ and $y_{2 c}$ be the course spacing and the wale spacing respectively in critical tensile state, and $D$ the yarn thickness in non-compressed state.

Dividing the unit structure of the front-bar yarn into two parts at point $N_{1}$, the lenghs of the sinker and the needle loops, $S_{f s}$ and $S_{f n}$, are given by

$$
\left.\begin{array}{c}
S_{f s}\left(\lambda_{s 1}, \lambda_{s 2}, D_{p f s}, D_{p f n}\right)=L_{1 f}\left(\lambda_{s 1}, \lambda_{s 2}, D_{p f n}\right) \\
\cdots A\left(\lambda_{s 1}, D_{p f n}\right)+C_{f}\left(D_{p f s}\right) \\
S_{f n}\left(\lambda_{s 1}, \lambda_{s 2}, D_{p f n}\right)=2 L_{2 f}\left(\lambda_{s 1}, \lambda_{s 2}, D_{p f n}\right) \\
\quad-B_{f}\left(D_{p f f}\right)
\end{array}\right\} \cdots \cdots \cdot(4)
$$

where $L_{1 f}$ and $L_{2 f}$ are lengths of the straight parts of the sinker and the needle loops respectively, and are calculated by

$$
\left.\begin{array}{l}
L_{1 f}\left(\lambda_{s 1}, \lambda_{s 2}, D_{p f n}\right) \\
=\sqrt{ }\left(\lambda_{s 1} y_{1 c}-D\right)^{2}+\lambda_{s 2}{ }^{2}\left(n_{f} y_{2 c}-D\right)^{2} \\
+A^{2}\left(\lambda_{s 1}, D_{p f n}\right)-2 D \\
L_{2 f}\left(\lambda_{s 1}, \lambda_{s 2}, D_{p f n}\right) \\
\therefore \sqrt{ }\left(\lambda_{s 1} y_{1 c}-D\right)^{2}+0.5 D^{2}\left(\lambda_{s 2}{ }^{2}+\lambda_{s 2}+1\right) \\
\quad\left(0.5 \lambda_{s 2}+1.5\right) D \cdot D_{p f n}+1.5 D_{p f n}^{2} \\
A\left(\lambda_{s 1}, D_{p f n}\right)=\left(1.5 D+D_{p f n}\right) D \\
/\left(\lambda_{s 1} y_{1 c}-D\right) \div A\left(\lambda_{s 1}, D_{p f s}\right)
\end{array}\right\} \cdots \cdots(5)
$$

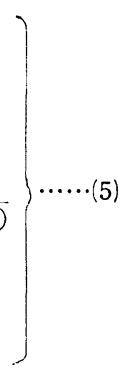

$B_{f}$ is the length $L_{1 f}$ subtracted from the yarn length in needle loop and $C_{f}$ is the length $L_{1 f}$ subtracted from the yarn in length sinker loop, and these are given by

$$
\begin{aligned}
& B_{f}\left(\lambda_{s 2}, D_{p f n}\right)=\pi\left(1.125 D_{p f n}+0.875 D\right)+\lambda_{s 2} D+0.5 D \\
& C_{f}\left(D_{p f s}\right)=\pi\left(0.875 D_{p f s}+0.625 D\right)+1.5 D
\end{aligned}
$$

Then, the stretch ratios of the sinker and the needle loop yarns, $\lambda_{y 1 f}$ and $\lambda_{y m f}$, are calculated by the following equation, instead of eqs.(15) and (16) appeared in the previous paper ${ }^{[1]}$.

$$
\left.\begin{array}{l}
\lambda_{y 1 f}=S_{f s} /\left\{L_{10 f} \div(1.5 \pi \cdots 0.5) D-A_{c}\right] \\
\lambda_{y m f}=S_{f n} /\left[2 L_{20 f}+(2 \pi+1.5) D\right]
\end{array}\right\} \cdots \cdots(6)
$$

where $L_{10 f}, L_{20 f}$ and $A_{c}$ are obtained as follows:

$$
\begin{aligned}
L_{10 f} & =\sqrt{\left(y_{1 c}-D\right)^{2}+\left(n_{f} y_{2 c}-D\right)^{2}+A_{c}^{2}} \\
L_{20 f} & =\sqrt{\left(y_{1 c}-D\right)^{2}}-5 D^{2} \\
A_{c} & =2.5 D^{2} /\left(y_{1 c}-D\right)
\end{aligned}
$$

As the tensile property of a yarn is a function of yarn stretch ratio, yarn tensions, $T_{1 f}$ and $T_{m f}$, of the sinker loop and the needle loop are obtained if the yarn stretch ratios, $\lambda_{y 1 f}$ and $\lambda_{y m f}$, are given respectively:

$$
\begin{aligned}
& T_{1 f}=f\left(\lambda_{y 1 f}\right) \\
& T_{m f}=f\left(\lambda_{y m f}\right)
\end{aligned}
$$<smiles>[Te][TeH]</smiles>

When a yarn under tension is wound around a rod having diameter $D$, the pressures $Q_{1_{f}}$ and $Q_{m f}$ acting on the contact area, due to yarn tensions $T_{1 f}$ and $T_{m f}$, can be calculated as

$$
\begin{aligned}
& Q_{1 f}=2 T_{1 f} / D \\
& Q_{m f}=2 T_{m f} / D
\end{aligned}
$$<smiles>CCC[Hg]</smiles>

Because the thickness $D_{p}$ under the pressure $Q$ can be experimentally measured, $D_{p f s}, T_{1 f}, D_{p f n}$ and $T_{m f}$ are obtained by the graphical method ${ }^{[1]}$, and the tensile loads

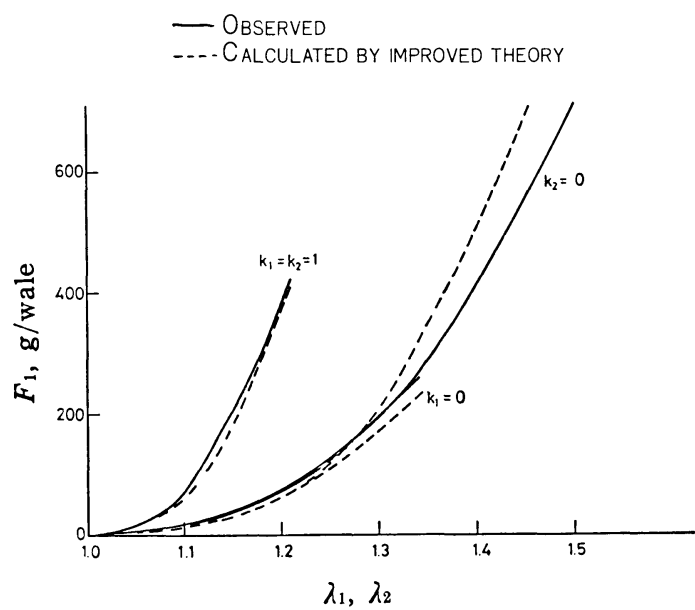

(a) $F_{1}$

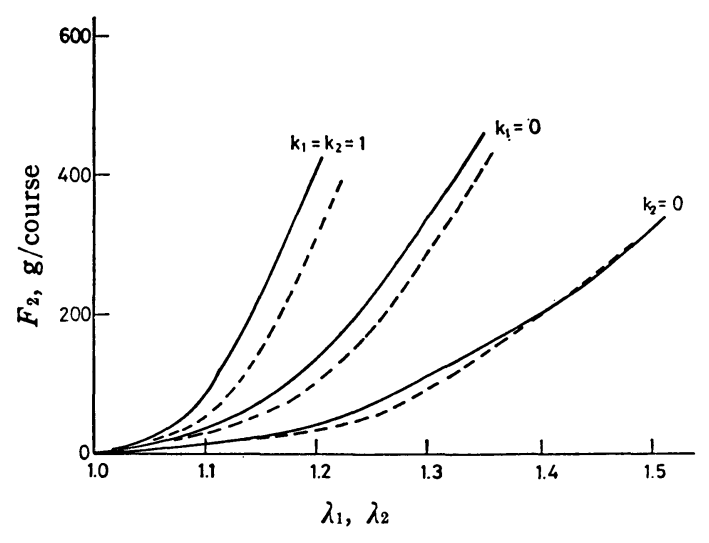

(b) $F_{2}$

Fig. 13 Comparison between between theoretical and experimental curves ( $\$ 5$ nylon 100 den sharkskin fabric) 


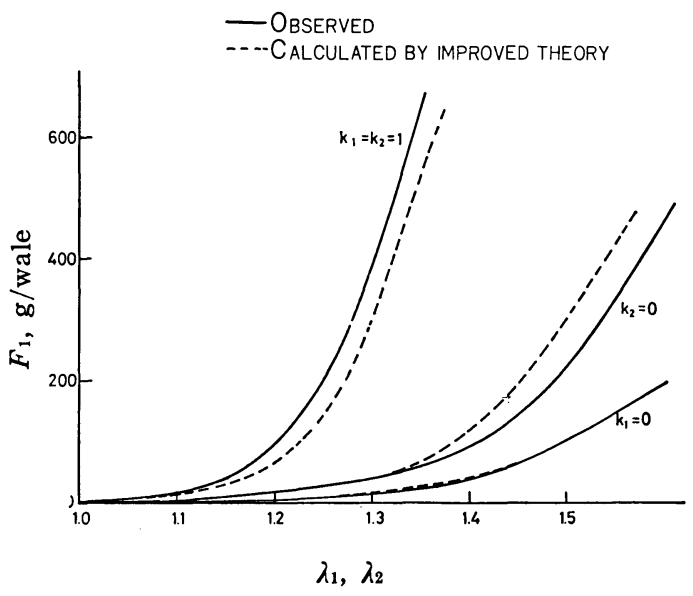

(a) $F_{1}$

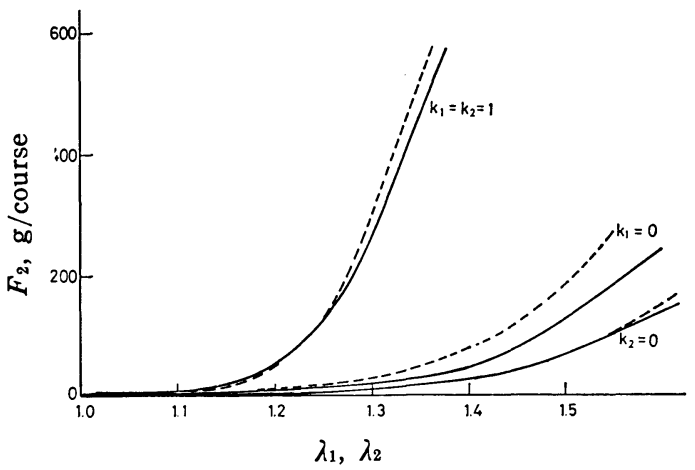

(b) $F_{2}$

Fig. 14 Comparison between theoretical and experimental curves ( $\$ 8$ cotton half fabric)

are calculated as a function of stretch ratios $\lambda_{s 1}$ and $\lambda_{s 2}$.

These improvements in the theory are done also with regard to the back-bar yarn in the same manner as done with the front-bar yarn as mentioned above.

\section{Comparison between the Experimental Value and the Theoretical Value}

The curves calculated by the improved theory are shown by dotted lines in Figs. 10 and 11. These figures show that the agreement between theoretical curves calculated by the improved theory and experimental curves is much better than that before the theory is improved. Some examples of specimens $\# 5$ and $\$ 8$ are shown in Figs. 13 and 14 .

Therefore, it is confirmed that by this improvements the theory has been made more useful for the practical design of almost all kinds of two bar tricot fabrics.

\section{Conclusion}

In the previous paper, a theoretical method for calculating the biaxial tensile property of two bar tricot fabrics was presented.

In this paper, the accuracy of this theory has been investigated by measuring the tensile property of some actual fabrics by means of a biaxial tensile tester, and comparing the measured values with the theoretical ones.

The result shows that the fabrics knitted of fine or lowbulky yarns provide a very good agreement between the theoretical and experimental results. For the fabrics knitted of thick or high-bulky yarns, however, there is some discrepancy between them in some biaxial deformation modes.

Two improvements have been done on this theory based on those observations; (1) the structure of the unit model has been corrected to be closer to the actual structure, and (2) the compressive deformations of yarns in the needie and the sinker loops are calculated separately.

These improvements have made the theory more useful for the practical design of almost all kinds of two bar tricot fabrics.

Grateful acknowledgment is due to members of Fiber Assembly Study Group in Department of Polymer Chemistry of Kyoto University for useful discussion, and also to K. Nakagawa for his help in the experiments.

The authors would like to express their thanks to Osaka Branch of Textile Research Institute, Osaka Prefecture, Toyo Spinning Co., Ltd. and Mitsubishi Rayon Co., Ltd. for supplying samp'es.

\section{Literature cited}

[1] Y. Yanagawa, S. Kawabata, H. Kawai; J. Text. Mach. Soc. Japan, English ed., 18, 55(1972)

[2] K. Sakaguchi, S. Kawabata, H. Kawai, N. Hazama; Material, 17, 365 (1968)

[3] Y. Yanagawa, S. Kawabata, K. Nakagawa, K. Toyama, H. Kawai; J. Text. Mach. Soc. Japan, English ed., 16, 126 (1970)

[4] Y. Yanagawa, S. Kawabata, K. Nakagawa, K. Toyama, H. Kawai; J. Text. Mach. Soc. Japan, English ed., 16, 216 (1970)

[5] Y. Yanagawa, S. Kawabata, H. Kawai; J. Text. Mach. Soc. Japan, English ed., 17, 15 (1971)

[6] S. Kawabata, M. Niwa, Y. Nanashima, H. Kawai; J. Text. Mach. Soc. Japan, 23, T223 (1970)

[7] M. Niwa, S. Kawabata, Y. Nanashima, H. Kawai; J. Text. Mach. Soc. Japan, 23, T120 (1970)

[8] S. Kawabata; Fiber Science and Industry, Japan, 1, 476 (1968) 